ÉGYPTE monde arabe

\section{Égypte/Monde arabe}

1 | 1999

L'expédition de Bonaparte vue d'Égypte

\title{
Agenda : Les manifestations commémoratives
}

Colloques, expositions, actualité éditoriale

\section{Ghislaine Alleaume}

\section{(2) OpenEdition}

Journals

Édition électronique

URL : https://journals.openedition.org/ema/773

DOI : $10.4000 /$ ema.773

ISSN : 2090-7273

Éditeur

CEDEJ - Centre d'études et de documentation économiques juridiques et sociales

Édition imprimée

Date de publication : 30 juin 1999

Pagination : 213-219

ISBN : 2-87027-773-3

ISSN : 1110-5097

Référence électronique

Ghislaine Alleaume, "Agenda : Les manifestations commémoratives », Égypte/Monde arabe [En ligne], 1 | 1999, mis en ligne le 08 juillet 2008, consulté le 07 juillet 2022. URL : http://

journals.openedition.org/ema/773 ; DOI : https://doi.org/10.4000/ema.773

Ce document a été généré automatiquement le 7 juillet 2022.

Tous droits réservés 


\title{
Agenda : Les manifestations commémoratives
}

\author{
Colloques, expositions, actualité éditoriale
}

\author{
Ghislaine Alleaume
}

1 Placée sous l'égide des deux ministères des Affaires étrangères et de la Culture et de la Communication pour la partie française, du ministère de la Culture pour la partie égyptienne, la célébration des relations franco-égyptiennes a donné lieu, durant l'année 1998, à une centaine de manifestations ${ }^{1}$. Elles se proposaient, sous le label des «Horizons partagés ", de dresser l'inventaire des "affinités électives» unissant les deux pays et de permettre «aux publics français et égyptien de mieux connaître la culture de l'autre, tant dans sa dimension historique et patrimoniale que dans sa dimension vivante $»^{2}$. En dépit de cette symétrie affichée, elles ont été beaucoup plus nombreuses en France qu'en Égypte. Plus diversifiées et mieux distribuées sur le territoire, elles ont aussi bénéficié d'un plus grand retentissement. Ce simple constat suffit à dire le décalage subsistant entre les deux pays dans leur rapport à la mémoire d'un événement, qu'on souhaitait ne pas évoquer, mais qui resta omniprésent.

2 En France, l'action des deux ministères de tutelle et de leurs organes exécutifs (l'Association française d'action artistique pour le ministère des Affaires étrangères et le département des Relations internationales pour le ministère de la Culture), fut relayée par un large partenariat: l'année franco-égyptienne reçut ainsi le concours de l'Institut du monde arabe et de la Ville de Paris, et surtout d'une trentaine de villes de province, de taille parfois fort modeste ${ }^{3}$, mais qui avaient, par l'un de leurs natifs ou par une collection de leur musée municipal, un lien quelconque avec l'Égypte. Il suffisait que Monge, Fournier, Vivant Denon, Jollois et quelques autres fussent bourguignons, pour que se tienne à Auxerre une exposition sur « La contribution des Bourguignons au rêve oriental ». Ailleurs, c'était moins à l'expédition de Bonaparte qu'à l'engouement égyptophile qu'elle suscita, ou aux très nombreux experts français qui furent au service des khédives et léguèrent à leur ville natale tout ou partie de leurs collections, que ces municipalités durent leur engagement dans la fête égyptienne. Parfois encore, ce fut l'idée d'une thématique particulière : celle du fleuve, par exemple, pour Toulouse qui 
choisit de célébrer les Musiques du Nil en invitant des troupes de musique traditionnelle égyptienne à son festival, organisé autour de la voie d'eau que forme la Garonne. Et un fleuve en valant un autre, le festival de la Garonne partagea une partie de sa programmation avec celui de Chalon-sur-Saône. La pluralité des lieux et la multiplicité des liens les unissant à l'Égypte donnèrent ainsi aux manifestations une belle diversité : de la bande dessinée au colloque, du film aux musiques traditionnelles, tous les supports et toutes les disciplines artistiques furent mobilisés.

3 En Égypte, le ministère de la Culture, représenté en tant que tel et par sept de ses services, n'eut pour tout partenaires que deux autres ministères, les Affaires étrangères et le Tourisme. L'extrême centralisation du gouvernement égyptien et le caractère très politique de la gestion territoriale, qui rend impossible toute forme de coopération décentralisée, contribuèrent sans doute à enfermer les manifestations dans des cercles trop étroits pour leur valoir l'adhésion d'un large public. Les sociétés savantes, les académies et les universités égyptiennes restèrent à l'écart du projet et toutes les manifestations furent concentrées à Alexandrie ou au Caire. Rosette, pourtant, aurait pu s'associer à Figeac pour fêter une pierre qui a au moins le mérite de mettre d'accord Français et Égyptiens, et Alexandrie accueillir, aussi bien qu'Ajaccio, la très belle exposition organisée par le musée Fesch autour de la Description de l'Égypte et des collections du consul Drovetti prêtées par le musée de Turin. Dans le déséquilibre qui entacha ce bicentenaire, entrent donc bien des éléments. L'inévitable différence des mémoires nationales n'est pas seule en cause : le rôle de l'État dans la construction du discours sur soi et l'espace consenti aux libertés citoyennes pesèrent aussi de tout leur poids.

Quels que soient les débats qu'elles suscitent, les commémorations ont toujours au moins le mérite de fournir l'occasion de quelques découvertes. L'année francoégyptienne ne fit pas exception à la règle et elle laisse derrière elle quelques réalisations durables. Selon une tradition bien française, les grandes expositions se sont encore une fois taillé la part du lion. Pour échapper aux pesanteurs de "l'incommémorable » expédition, les organisateurs avaient conjointement choisi de mettre l'accent sur des moments prestigieux de l'histoire culturelle des deux pays partenaires : la France fut ainsi invitée à découvrir l'Égypte fatimide ${ }^{5}$ et l'Alexandrie hellénistique ${ }^{6}$ tandis qu'elle offrait aux Égyptiens l'un des âges d'or de la peinture française, de Corot à Picasso ${ }^{7}$. C'était, dans tous les cas, retenir des périodes marquées par le cosmopolitisme de la production culturelle et artistique: les chefs-d'œuvre présentés dans ces trois expositions, qui formaient la part la plus officielle des manifestations, plaidaient tous pour l'ouverture au monde, le mélange des peuples, des langues, des cultures et des influences.

De l'expédition, lorsqu'on en parla, on retint surtout, comme on pouvait l'attendre, l'œuvre scientifique. À Paris, le Muséum d'histoire naturelle, qui conserve une large part des collections de l'expédition, célébra l'aventure savante en une exposition ${ }^{8}$ à la fois érudite et didactique, où l'on pouvait découvrir, pour la première fois sortis des réserves, les documents originaux collectés par les botanistes, zoologues et autres savants de l'expédition. Là se consignait au jour le jour, et bien en amont de la Description de l'Égypte, le grand inventaire qui fonde encore la fascination exercée sur l'imagination populaire par la campagne d'Égypte. On en voyait en quelque sorte la face cachée, le laboratoire plus que le résultat, l'invention bricoleuse plus que la méthode. À Ajaccio, le musée Fesch prit une option similaire dans une exposition qui avait pour 
thème central la Description de l'Égypte ${ }^{9}$. Élargissant fort pertinemment leur lecture de l'œuvre, les organisateurs la placèrent entre le remarquable Plan terrier de la Corse, réalisé par Testevuide et qui allait servir de modèle à l'Atlas de l'Égypte, les superbes collections constituées sous le règne de Muhammad 'Alî par le consul Drovetti, et l'expédition franco-toscane de 1829-1830. Ils offraient ainsi à l'amateur éclairé une occasion unique de lire en continu une séquence centrale de la formation des savoirs sur l'Égypte.

6 Deux autres expositions entraînaient le public à la découverte d'une Égypte un peu postérieure à celle de l'expédition. La bibliothèque municipale de Marseille présentaient les travaux égyptiens de l'ingénieur et architecte Pascal Coste ${ }^{10}$, que l'on ne connaît guère, en Égypte, que pour son livre sur Les monuments de l'Art Arabe du Kaire, mais qui a aussi légué à sa ville natale un ensemble extraordinaire de projets, de dessins et d'aquarelles, rarement exposés en raison de leur fragilité. La Roche sur Yon fit également honneur à l'un de ses enfants, l'architecte Ambroise Baudry ${ }^{11}$, qui exerça au Caire de 1871 à 1886 et fut un temps l'architecte en chef des palais khédiviaux. Outre son intérêt intrinsèque - exhumer l'œuvre d'un artiste oublié - cette exposition avait aussi celui d'être le résultat d'une recherche ad hoc conduite à l'occasion de l'année franco-égyptienne. Elle était, en ce sens, exemplaire du parti que l'on peut tirer d'une commémoration.

7 De ces quatre expositions plus spécifiquement tournées vers l'histoire de l'Égypte, aucune ne traversa la Méditerranée. Les découvertes qu'elles invitaient à faire furent réservées au seul public français. Et c'est bien dommage, car il y avait là matière à un vrai partage. Ces thématiques étaient celles qui étaient le plus susceptibles de toucher un large public égyptien, avide, de surcroit, d'une pédagogie de l'image qui lui fait encore grandement défaut. Les historiens, notamment, auraient eu la chance d'avoir ainsi accès à des documents dont ils ignorent presque tout. Privés du plaisir visuel que donne la visite d'une exposition, les Égyptiens devront s'en remettre aux seuls catalogues ou livres d'art qui accompagnaient, fort heureusement, la plupart de ces manifestations. Car le dernier élément du succès des festivités françaises tint à la mobilisation massive des scientifiques comme des éditeurs. L'année franco-égyptienne fut d'abord le prétexte à plusieurs colloques, bien moins voués à la célébration événementielle que soucieux de faire le point sur quelques questions d'historiographie. Le cadre conceptuel en avait été esquissé par une grande enquête réalisée, quelques années plus tôt, dans le cadre d'un appel d'offres lancé par le ministère de la Recherche sur «La méditerranée : échanges et affrontements", et dont les premiers résultats parurent précisément en cette année 1998. La réflexion amorcée par les coordinateurs de ce programme sur les trois expéditions scientifiques et militaires lancées par la France dans la première moitié du XIX siècle, en Égypte, en Morée et en Algérie, permettait de redéfinir sous un angle radicalement nouveau l'examen des relations entre science et guerre, savoir et pouvoir ${ }^{12}$. Un peu fortuitement, L'Invention scientifique de la Méditerranée préparait ainsi la voie aux divers « colloques du Bicentenaire ».

8 Le plus solennel fut celui organisé sous le patronage des cinq académies, avec le concours du Muséum d'histoire naturelle, en l'honneur de l'éphémère institut fondé au Caire par Bonaparte. Sous un titre d'apparence fort sage - «L'expédition d'Égypte, une entreprise des Lumières $\|^{13}-$, ce colloque, organisé par un historien des sciences, poursuivait la déconstruction critique amorcée par l'Invention scientifique. Il s'ouvrait par une très belle communication de Marie-Noëlle Bourguet sur « science et guerre » et 
l'on ne peut que regretter, une fois encore, que de telles approches aient été développées sans les Égyptiens. Plusieurs historiens avaient été pressentis, mais soit par conviction personnelle, soit parce que les polémiques suscitées en Égypte par la commémoration faisaient apparaitre comme traîtres à l'idée nationale tous ceux qui acceptaient d'y participer, ils avaient préféré se désister. Le colloque d'Aix-en-Provence sur l'Égypte khédiviale ${ }^{14}$ fit exception, en raison sans doute de la personnalité de l'organisateur : grande figure de l'histoire ottomane, très largement lu et étudié dans le monde arabe, André Raymond était trop peu suspect de pouvoir cautionner une entreprise « coloniale » pour susciter autant de défiance. Les médiévistes égyptiens, en revanche, purent, à l'abri des querelles, participer au colloque international organisé par la fondation Singer-Polignac sur l'Égypte Fatimide ${ }^{15}$, le premier du genre et qui, réunissant les meilleurs spécialistes mondiaux de la période, laisse un ouvrage qui fera sans doute longtemps référence. D'autres rencontres, encore, accompagnèrent les expositions. Ce fut le cas du colloque d'Ajaccio sur Bonaparte et les îles méditerranéennes ${ }^{16}$, ou de celui organisé aux Invalides par le Musée militaire.

L'action des universitaires, enfin, fut relayée par celle des éditeurs. Parmi tous les titres de circonstance, trois d'entre eux placent des jalons durables dans la bibliographie sur l'expédition d'Égypte. Dans son Égypte, une aventure savante ${ }^{17}$, Yves Laissus, qui fut l'un des organisateurs de l'exposition du Muséum d'histoire naturelle, dresse un nouveau bilan, très informé, de l'histoire scientifique de l'expédition. Cet ouvrage, qui a de surcroît le mérite d'être superbement illustré, constituera pour longtemps la synthèse de référence sur l'aspect à la fois le plus populaire et le plus mal connu de la campagne d'Égypte. Chacun à sa manière, Patrice Bret et André Raymond ont, eux, pris le parti de donner une vision plus égyptienne des trois années de l'occupation française: le premier en relevant le difficile défi d'esquisser une histoire de la vie quotidienne en Égypte au temps de l'expédition ${ }^{18}$, dans un petit livre aussi bien documenté qu'agréablement rédigé ; et le second en inversant la perspective dominante pour donner, dans toute la mesure du possible, la parole aux Égyptiens, en une relecture de l'événement fondée sur l'examen systématique des sources arabes contemporaines ${ }^{19}$. Pris ensemble, ces deux derniers livres, qui semblent se répondre, offrent au lecteur cultivé un tableau mis à jour et nuancé d'un épisode à la fois séduisant et douloureux de l'histoire des relations franco-égyptiennes. Parallèlement à ces travaux de spécialistes, parurent aussi des ouvrages de vulgarisation, dont les plus réussis sont celui de Robert Solé, qui réunit en un volume des articles parus durant tout l'été 1998 dans le journal Le Monde ${ }^{20}$, et le petit fascicule paru dans la collection «Découvertes », sous la plume de Laure Murat et Nicolas Weill ${ }^{21}$. Pour ne pas être en reste avec la modernité, la commémoration fut aussi l'occasion de l'édition de plusieurs CD-rom, dont le plus abouti s'appuyait sur l'exposition du Muséum ${ }^{22}$.

10 En Égypte, les polémiques suscitées par ce que l'on percevait comme la commémoration d'une expédition coloniale ont beaucoup limité la portée des festivités. Une vingtaine de manifestations seulement se tinrent au Caire ou à Alexandrie et, pour la plupart, elles avaient été pensées et préparées en France. La programmation musicale, notamment, passionnante pour le mélomane averti, était sans doute trop difficile pour trouver un public dépassant quelques dizaines d'initiés ${ }^{23}$. Seule fit exception la très belle exposition organisée avec le concours du musée d'Orsay « De l'impressionisme à la modernité. Un siècle de peinture française de Corot à Picasso »: par la qualité des œuvres retenues et l'originalité des choix - souvent inattendus, voire même paradoxaux -, elle offrait à l'amateur égyptien une confrontation directe, didactique et 
novatrice à la fois, avec les courants majeurs de l'art contemporain. Cette entreprise initiatique était en outre servie par un beau catalogue bilingue ${ }^{24}$. Les chercheurs, on l'a $\mathrm{vu}$, avaient, dans leur majorité, pris le parti de rester à l'écart. L'activité savante fut donc réduite au minimum. Un seul colloque se tint au Caire. Il fut organisé, il est vrai, par une vénérable société savante, la Société égyptienne des études historiques, et accueilli dans le décor prestigieux de la très belle bibliothèque du Caire. Il eut du succès, suscita de vraies et belles controverses, mais, hélas, n'apporta guère de contributions nouvelles à la recherche historique. La polémique, qui obligeait à débattre moins d'histoire qu'à se lancer dans des surenchères nationalistes, tua décidément le débat d'idées. Dans l'actualité éditoriale, L'expédition d'Égypte au tribunal de l'histoire de Laïla Enan est le seul titre dont la parution ait été directement liée à l'événement. En deux petits volumes publiés en format de poche et destinés au public le plus large, l'auteur - qui fut l'un des plus vifs adversaires de la commémoration offrait une critique construite et bien informée d'une historiographie apologétique, égyptienne aussi bien qu'européenne, déterminée à ne retenir de l'aventure de Bonaparte que la dimension idéelle de l'inventaire encyclopédique. En comparaison, le livre de Hilmî Namnam, Les Égyptiens et l'expédition de Bonaparte, paru un peu plus tôt, faisait figure de pâle ouvrage de circonstance, anticipant sur une polémique dont bien des éléments étaient convenus.

11 Sur un versant plus académique, l'année franco-égyptienne fut, malgré tout, l'occasion de l'édition ou de la réédition d'un certain nombre de textes fondamentaux. Le plus remarquable est une traduction arabe d'un manuscrit ottoman écrit par 'Izzat effendi al-Dârindây, l'historiographe du grand-vizir Yûsuf Ziyâ pacha : repris d'une thèse de magistère, le livre de Gamâl 'Abd al-Ghânî offre aux historiens une source nouvelle et qui a le grand mérite de donner le point de vue des Ottomans, éternels oubliés d'un affrontement que l'on continue de voir comme trop exclusivement franco-égyptien. 'Abd al-Râziq 'Îsâ et 'Imâd Hilâl offraient, eux, une nouvelle édition critique du Mazhar al-taqdîs de Gabartî, la plus violemment anti-française des trois chroniques de l'occupation que nous a laissées le grand historien. Leur contribution sera également précieuse aux historiens mais, dans ce domaine, on peut regretter, avec André Raymond, que l'on n'ait pas opté pour une réédition en parallèle des trois textes. Une autre édition de la même œuvre fut, au contraire, publiée presqu'au même moment avec un fort parti d'apologétique musulmane, par un licencié de sciences politiques fort peu informé des règles de l'établissement critique d'un texte historique, mais néanmoins assez audacieux pour estimer pouvoir attribuer l'œuvre à la double plume de Gabartî et de Hasan al-'Attâr ${ }^{25}$. La grande chronique de Gabartî, les «Merveilles historiques " ('Ajẩib al-âthâr) fut elle aussi rééditée par 'Abd al-'Azîz Gamâl al-dîn, avec un parti historiograhique curieux, puisqu'il mêle au texte original d'autres documents censés l'avoir inspiré. Enfin, une publication exhaustive des documents d'archives relatifs à l'expédition, conservés en Égypte, devait être préparée à l'université de 'Ayn Shams par un groupe de jeunes chercheurs placés sous la tutelle du professeur 'Abd al-'Azîz Nawwâr. Elle n'a pas encore vu le jour, mais le projet n'en est pas abandonné. Pour de multiples raisons, il a simplement "manqué » le rendez-vous de l'année franco-égyptienne.

12 Le bilan, au total, est assez mince et dit bien l'inégal intérêt porté à ce bicentenaire de part et d'autre de la Méditerranée. Nous avons pris le parti de joindre aux comptes rendus de ces parutions récentes, quelques présentations succinctes d'ouvrages plus anciens et de thèses égyptiennes, publiées ou restées inédites, généralement ignorées 
de la recherche internationale. L'ensemble donnera au lecteur francophone un bon résumé de l'état de la question, telle qu'elle est formulée au Caire. On verra, à la lecture de ces notes, à quel point l'histoire de l'expédition d'Égypte reste grevée d'une double contrainte : celle que crée l'impossibilité pour les chercheurs égyptiens d'accéder aux archives françaises, infiniment plus riches et plus nombreuses; et celle qu'imposent les convulsions de la construction de la mémoire collective.

\section{NOTES}

1.Pour un historique de la mise en place de cette année franco-égyptienne, on pourra se reporter au mémoire de Christelle Crouzet «France-Égypte, Horizons partagés : une étape dans les échanges culturels franco-égyptiens? ", mémoire de DESS en développement et coopération, Paris I, dept. de sciences politiques, 1997, 60 p., dactylographié.

2.Jean-Jacques Aillagon, commissaire général pour la France, présentation du dossier de presse « France-Égypte, Horizons partagés », Paris, 1997, dactylographié, 150 p. 3.La liste des trente-deux villes qui furent les partenaires officiels des « Horizons partagés » est donnée dans le dossier de presse déjà cité. On y trouve Saint-Céré, Souillac et La Roche-sur-Yon aussi bien que de grandes capitales régionales, comme Bordeaux, Marseille ou Toulouse.

4.Selon l'expression de Mona Ozouf, 1789, La commémoration, Paris, Le débat/Folio Histoire, Gallimard, 1999, p. 16.

5.Trésors fatimides du Caire, Institut du monde arabe, avril-août 1998. Catalogue publié sous le même titre, Paris, IMA, 1998.

6.La Gloire d'Alexandrie, Paris, Petit Palais, mai-juillet 1998.

7.De l'impressionnisme à la modernité, Un siècle de peinture française, Le Caire, Galerie du Nil, avril-juillet 1998.

8.Il y a 200 ans, les savants en Égypte, Paris, Muséum d'histoire naturelle, mars-juin 1998. Catalogue édité sous le même titre, Paris, Nathan, 1998.

9.La Description de l'Égypte, reflets d'une civilisation, Ajaccio, musée Fesch, avril-août 1998. L'exposition empruntait au musée de Turin des éléments de la collection Drovetti et à celui de Florence, les documents de l'expédition franco-toscane.

10.Pascal Coste, Toutes les Égypte, bibliothèque municipale de Marseille, mai-juin 1998. Catalogue édité sous le même titre par Dominique Jacobi (éd.), Marseille, Éditions Paranthèses et Bibliothèque municipale de Marseille, 1998, $250 \mathrm{p}$.

11.L'Égypte d'un architecte, Ambroise Baudry (1838-1906), La Roche-sur-Yon, avril-juin 1998. L'exposition tourna ensuite dans diverses autres villes, jusqu'en mai 1999. Il n'y eut pas de catalogue à proprement parler, mais l'enquête qui permit la réalisation de cette exposition donna lieu à un ouvrage collectif, publié sous le même titre, Paris, Éditions Somogy, 1998, 168 p. et 120 illustrations.

12.Marie-Noëlle Bourguet, Bernard Lepetit, Daniel Nordman, Maroula Sinarellis, L'invention scientifique de la Méditerranée, Égypte, Morée, Algérie, Paris, Éditions de l'École des hautes études en sciences sociales, 1998, 325 p. 
13.L'expédition d'Égypte, une entreprise des Lumières, Institut de France et Muséum d'histoire naturelle, Paris, 8-10 juin 1998. Les Actes sont à paraître dans les Archives de l'Académie des Sciences, 1999.

14.La France et l'Égypte à l'époque des vice-rois (1805-1882), Aix-en-Provence, 5-7 juillet 1998.

15.L'Égypte fatimide : son art et son histoire, Paris, Université de Paris IV, 29-30 mai 1998. Les Actes sont à paraître aux Presses de l'Université de Paris-Sorbonne, 1999.

16.Bonaparte, les îles méditerranéennes et l'appel de l'Orient, Ajaccio, musée Fesch, 29-30 mai1998. Il a été publié dans les Cahiers de la Méditerranée, « Bonaparte, les îles méditerranéennes et l'appel de l'Orient », Actes du colloque d'Ajaccio, 29 et 30 mai 1998, n 57, décembre 1998, 334 p.

17.Yves Laissus, L'Égypte, une aventure savante, Paris, Fayard, 1998.

18.Patrice Bret, L'Égypte au temps de l'Expédition de Bonaparte, Paris, Hachette, coll. « La vie quotidienne ", 1998, $340 \mathrm{p}$.

19.André Raymond, Égyptiens et Français au Caire, 1798-1801, Le Caire, IFAO, 1998, 391 p. 20.Robert Solé, Les savants de Bonaparte, Paris, Seuil, 1998, 251 p.

21.Laure Murat et Nicolas Weill, L'expédition d'Égypte : le rêve oriental de Bonaparte, Paris, Gallimard, coll. « Découvertes », 1998.

22.Réalisé par Olivier Nolin, Paris, Arte et Montparnasse éditions, 1998. Du même auteur, est également paru un petit livre d'accompagnement, Bonaparte et les savants français en Égypte, 1798-1801, Arte éditions et Mille et une Nuits, Paris, 1998, 143 p. 23.Le Festival Massenet et l'Opéra du Caire avaient monté ensemble Thaïs de Jules Massenet (décembre 1997) et l'Orchestre symphonique de l'Opéra du Caire, Le désert de Félicien David, également présenté à Figeac.

24.De l'impressionnisme à la modernité, Le Caire, AFAA, 1998, 228 p.

25.'Abd al-Rahmân al-Gabartî et Hasan al-'Attâr, Mazhar al-taqdîs bi-dhahâb dawlat alfaransîs, édité par Ahmad 'Abduh cAlî, Le Caire, Maktabat al-âdâb, 1998, 294 p. Cet ouvrage n'est pas recensé ici.

\section{INDEX}

Mots-clés : historiographie 